\title{
Bispectral index analysis in general anesthesia: An observational study
}

\author{
G V Rao ${ }^{1}$, Kiran Chand $\mathrm{N}^{2, *}$, A. Srinivasa Murthy ${ }^{3}$, Balabhaskar $\mathrm{S}^{4}$ \\ ${ }^{\mathbf{1}}$ Associate Professor, ${ }^{2}$ Assistant Professor, ${ }^{4}$ Professor, Dept. of Anesthesiology, Vijayanagar Institute of Medical Sciences, \\ Ballari Karnataka, ${ }^{3}$ Professor, Dept. of Anesthesiology, Novodaya Medical College, Raichur, Karnataka, India
}

\author{
*Corresponding Author: \\ Email: nkiranchand@yahoo.com
}

Received: $5^{\text {th }}$ March, 2018

Accepted: $9^{\text {th }}$ April, 2018

\begin{abstract}
Introduction: Bispectral index monitoring (BIS) is one of the major techniques used in monitoring depth of anesthesia. The present study was carried out to observe the changes in BIS values vis-a-vis the changes in hemodynamic parameters during the standard general anaesthesia practices in our institute.

Materials and Methods: This prospective observational study was carried out in 50 ASA I/II elective surgeries in adults under general anesthesia. Conditions and drugs likely to interfere with BIS values were excluded. Anesthesia protocol was kept uniform. Patients were induced with IV propofol $2 \mathrm{mg} / \mathrm{kg}$, IV pentazocine $0.5 \mathrm{mg} / \mathrm{kg}$ and IV vecuronium $0.1 \mathrm{mg} / \mathrm{kg}$. Patients were intubated with appropopriate size endotracheal tube and oxygen, nitrous oxide, halothane and vecuronium were used for maintanence of anesthesia. Patients were reversed with IV neostigmine $0.05 \mathrm{mg} / \mathrm{kg}$ and I V Atropine $20 \mathrm{mcg} / \mathrm{kg}$. The BIS values, hemodymanic changes, recovery characteristics (including awareness) were noted. Correlation between BIS scores and hemodynamic parameters was assessed.

Results: There were predictable changes in BIS scores with the standard anesthesia technique followed in the present study. Similarly, hemodynamic changes were also on the expected lines during the course of anesthesia. However, the correlation between BIS score and hemodynamics could not be established.

Conclusion: Bispectral Index Monitor is a useful tool to assess the depth of anesthesia in standard anesthetic management. Alterations in anesthetic management and drug dosages can be guided by BIS scores. Cost factor and the need to use updated and refined versions of the software are the possible limitations.
\end{abstract}

Keywords: Electroencephalography, Consciousness monitors, Anesthesia, General, Hemodynamics.

\section{Introduction}

Intraoperative awareness is a major medico-legal liability to the anaesthesiologists and can lead to postoperative psychosomatic dysfunction in the patient, and therefore should be avoided at all costs. ${ }^{1,2}$ Intraoperative monitoring of the anaesthesia depth is important for the prevention of this problem. ${ }^{3}$

Various electroencephalographic (EEG) variables have been studied as possible indicators of anaesthetic adequacy like the power spectral analysis, spectral edge frequency, bispectral index, entropy, cerebral state monitor and evoked responses. ${ }^{4}$

Bispectral analysis (BIS) of the $\mathrm{EEG}^{5}$ is a signal processing technique that has been proposed as a pharmacodynamic measure of anaesthetic effects on the central nervous system. This method decompresses the EEG and quantifies the level of synchronization in the signal, along with the traditional amplitude and frequency variables, thereby providing a more complete description of complex EEG patterns.

BIS monitoring has some potential advantages over conventional intermittent techniques of patient assessment. Conventional assessment involves patient stimulation at frequent intervals to determine the level of consciousness, requiring patient co-operation and is subject to testing fatigue. Such limitations are overcome by using the bispectral index which is a non-invasive technique, wherein electrodes are applied over the forehead and BIS scores are displayed continuously and objectively in the monitor which indicate the level of consciousness.

Hence, the present study aims to observe BIS scores continuously in 50 patients in whom general anaesthesia is administered with propofol along with a standardized anaesthetic regime and correlate the bispectral index findings with various events in the intra-operative period and observe for awareness at 1 hour and 24 hours postoperatively.

\section{Materials and Methods}

The study was conducted in Department of Anaesthesiology, Vijayanagara Institute of Medical Sciences (VIMS), Bellary during the period from 1-122008 to 31-11-2009. Fifty patients of 18 to 60 years of age of ASA Grade I and II were selected for study after ethical committee approval. Informed consent was obtained from each patient. Patients undergoing elective general surgical procedures expected to last at-least one hour under general endotracheal anaesthesia such as laparotomy, cholecystectomy, hemithyroidectomy procedures were included in the study.

Patient who did not consent to the study, patients with known cardiac, renal, hepatic, neurological disorders, or any serious medical condition that would interfere with cardiovascular response assessment and use of benzodiazepines, anticonvulsants, alcohol, 
opioids or other psychotropic drugs (chronically or within 24 hours before the induction of anaesthesia) were excluded from the study.

A detailed pre-anaesthetic evaluation was done and the patients underwent routine investigations such as haemoglobin, total and differential count, urine routine, blood sugar, blood urea, serum creatinine, and special investigations were done as per the indication.

Standard NPO protocols were followed. On arrival in the operating room, routine anaesthetic drill was conducted and pre-induction monitors - pulse oximeter, non-invasive blood pressure (NIBP) and electrocardiogram (ECG) were connected. Following parameters were noted - systolic blood pressure (SBP), diastolic blood pressure (SBP) and mean arterial blood pressure (MBP), heart rate (HR), oxygen saturation $\left(\mathrm{SpO}_{2}\right)$. Intravenous (IV) access was established with 18 G IV cannula and an infusion of Ringer lactate started. After skin preparations, BIS electrodes (Aspect ${ }^{\circledR}$ Adult BIS Quatro electrodes, USA) were placed on the forehead and temple using a frontal - temporal montage and connected via module (version 1.0) to Beneview T5 ${ }^{\circledR}$ Multiparameter Patient Monitor (Shenzhen Mindray Bio-Medical electronics Co., LTD, China). BIS score was displayed on the monitor. The EEG was recorded continuously beginning before the induction of anaesthesia until patients were awake and responding to verbal commands after extubation. Patients were premedicated with inj. atropine $0.6 \mathrm{mg}$, and inj. pentazocine $0.5 \mathrm{mg} / \mathrm{kg} \mathrm{IV}$, and pre-oxygenated for $3 \mathrm{~min}$ with $100 \% \mathrm{O}_{2}$. IV induction was done with inj. propofol $2 \mathrm{mg} / \mathrm{kg}$ and inj. vecuronium $0.1 \mathrm{mg} / \mathrm{kg}$ using Bain circuit. Anaesthesia was maintained with oxygen, nitrous oxide $(33 \%, 66 \%)$, controlled ventilation along with halothane $0.5-1 \%$. Dose adjustment of anaesthetic agents to age, surgical and pathological condition of the patient was made accordingly. Post-induction monitoring included end-tidal carbon-dioxide. Any obvious changes in HR and BP necessitating supplementation with analgesics, hypnotics, and antihypertensive medications were carried out and recorded. Descriptions of all intra-operative events, along with measures taken to rectify them were recorded. Patient were reversed at the end of surgical procedure with inj. neostigmine $0.05 \mathrm{mg} / \mathrm{kg}$ and inj. atropine 0.02 $\mathrm{mg} / \mathrm{kg}$. Patients were extubated after adequate recovery from neuromuscular blockade and were observed for 20 min following extubation. All patients were interviewed for recall half an hour post-extubation and on the first post-operative day. Following questions were asked to enquire about awareness and recall in immediate postoperative period and on the first post -operative day:

1. What was the last thing you remembered before going to sleep?

2. What was the first thing you remembered on waking?
3. Do you remember anything between going to sleep and waking?

4. While you were sleeping during the operation, did you dream?

Bispectral index along with haemodynamics parameters were recorded before induction, $1 \mathrm{~min}$ and 3 min after induction with propofol, $30 \mathrm{secs}, 1 \mathrm{~min}, 3 \mathrm{~min}$, and 5 minutes after laryngoscopy and intubation, and 1,3,5,10 minutes after incision and every 5 min thereafter until skin closure. Post extubation, BIS score and haemodynamics were noted after 1,5,10 and 15 minutes. Primary objective of the study was to observe BIS value to assess the level of consciousness and depth of anaesthesia throughout the period of balanced general anaesthesia. Secondary objective was to simultaneously correlate the observed BIS scores with hemodynamic changes.

The statistical software namely SAS ${ }^{\circledR}$ 9.2, SPSS $^{\circledR}$ 15.0, Stata 10.1, MedCalc ${ }^{\circledR}$ 9.0.1, Systat $^{\circledR} 12 / 0$ and R environment ${ }^{\circledR}$ ver. 2.11 .1 were used for the analysis of the data and Microsoft ${ }^{\circledR}$ word and Excel have been used to generate graphs, tables etc.

Descriptive statistical analysis has been carried out in the present study. Results on continuous measurements are presented on Mean \pm standard deviation (SD) and results on categorical measurements are presented in number (\%). Significance is assessed at $5 \%$ level of significance.

The sample size was estimated by consulting Biostatistician and the information for samples size estimation taken from previous study. The sample size of 50 was enough to maintain the power of study at-least $80 \%$. Statistical software SAS $^{\circledR} 9.2$ was used to find the Sample size estimation.

\section{Results}

This prospective observational study was carried out on 50 patients by observing the depth of anaesthesia using the bispectral index monitor throughout the general anaesthetic technique. Procedures included were modified radical mastectomy, hemithyroidectomy, laparotomy- gastrojejunostomy and truncal vagotomy, appendicectomy, hernioplasty and superficial parotidectomy.

BIS scores showed changes at various changes in anaesthesia. On induction, BIS decreased to 45, increased to 59 by $3^{\text {rd }}$ min of intubation. On incision, the score increased to 62. At closure it increased to 78. Post extubation, a score of 93 was achieved in 15 min (Table $1)$. 
Table 1: Changes in bispectral index throughout the procedure

\begin{tabular}{|l|c|c|c|}
\hline \multirow{2}{*}{ Time } & \multicolumn{2}{c|}{ BIS index } & \multirow{2}{*}{ p-value } \\
\cline { 2 - 3 } & Mean & S.D & \\
\hline Basal & 96.34 & 1.70 & $0.000(<0.05)$ \\
\hline Induction $-3 \mathrm{~min}$ & 45.48 & 10.31 & $0.000(<0.05)$ \\
\hline Intubation-30 sec & 51.66 & 12.44 & $0.000(<0.05)$ \\
\hline Intubation-3 min & 59.08 & 9.82 & $0.000(<0.05)$ \\
\hline Incision $-1 \mathrm{~min}$ & 62.64 & 10.48 & $0.000(<0.05)$ \\
\hline Incision $-3 \mathrm{~min}$ & 62.68 & 10.06 & $0.000(<0.05)$ \\
\hline Closure $-5 \mathrm{~min}$ & 78.64 & 5.81 & $0.000(<0.05)$ \\
\hline Extubation $-1 \mathrm{~min}$ & 87.48 & 5.27 & $0.000(<0.05)$ \\
\hline Extubation $-15 \mathrm{~min}$ & 93.24 & 2.82 & \\
\hline
\end{tabular}

Table 2: Comparison of age and BIS in different age groups

\begin{tabular}{|l|c|c|c|}
\hline \multirow{2}{*}{ BIS } & \multicolumn{3}{|c|}{ Age in years (Mean \pm SD) } \\
\cline { 2 - 4 } & $18-30$ & $31-50$ & $>50$ \\
\hline Basal & $96.83 \pm 1.34$ & $96.48 \pm 1.3$ & $95.22 \pm 2.73$ \\
\hline Induction 3 min & $45.17 \pm 8.24$ & $45.72 \pm 9.29$ & $45.11 \pm 15.98$ \\
\hline Intubation 30 sec & $50.33 \pm 8.16$ & $52.28 \pm 12.74$ & $51.44 \pm 16.82$ \\
\hline 1 min & $54.67 \pm 7.24$ & $56.03 \pm 11.6$ & $52.44 \pm 11.17$ \\
\hline 3 min & $60.17 \pm 7.63$ & $60.17 \pm 9.28$ & $54.11 \pm 13.23$ \\
\hline Incision 1 min & $61.25 \pm 9.19$ & $62.45 \pm 11.4$ & $65.11 \pm 9.55$ \\
\hline 3 min & $62.67 \pm 8.49$ & $62.31 \pm 10.74$ & $63.89 \pm 10.73$ \\
\hline 5 min & $74.83 \pm 5.22$ & $72.59 \pm 7.93$ & $73.22 \pm 6.65$ \\
\hline Closure 5 min & $80.42 \pm 6.36$ & $77.38 \pm 5.54$ & $80.33 \pm 5.45$ \\
\hline Extubation 0 min & $86.67 \pm 4.38$ & $87.52 \pm 5.49$ & $88.44 \pm 6.04$ \\
\hline 5 min & $89.5 \pm 5.05$ & $89.66 \pm 3.95$ & $90.33 \pm 4.77$ \\
\hline 10 min & $90.25 \pm 3.93$ & $91.45 \pm 3.49$ & $91 \pm 4.15$ \\
\hline 15 min & $93.33 \pm 2.61$ & $93.38 \pm 2.6$ & $92.67 \pm 3.91$ \\
\hline
\end{tabular}

There were no significant differences between the age groups, except in one case where there was an exaggerated fall in BIS value to 22 on induction (Table 2).

Table 3: Showing heart rate changes during various stages

\begin{tabular}{|l|c|c|c|}
\hline \multirow{2}{*}{\multicolumn{1}{c|}{ Time }} & Mean & S.D & \multirow{2}{*}{ p-value } \\
\cline { 2 - 3 } & 85.02 & 10.57 & \\
\hline Basal & 99.16 & 15.87 & $0.000(<0.05)$ \\
\hline Induction 3 min & 104.34 & 16.07 & $0.000(<0.05)$ \\
\hline Intubation 30 sec & 102.48 & 16.61 & $0.000(<0.05)$ \\
\hline Intubation 3 min & 97.14 & 15.24 & $0.000(<0.05)$ \\
\hline Incision 1 min & 95.56 & 13.93 & $0.000(<0.05)$ \\
\hline Incision 3 min & 92.36 & 16.42 & $0.009(<0.05)$ \\
\hline Closure 5 min & 102.40 & 18.95 & $0.000(<0.05)$ \\
\hline Extubation 1 min & 85.80 & 18.99 & 0.793 \\
\hline Extubation 15 min & \multicolumn{2}{c|}{} &
\end{tabular}

There was fall in BIS while the HR increased at induction, at intubation both the HR and BIS increased. In the maintenance phase both the HR and BIS were well maintained throughout except in a case where there was high BIS score with normal HR (Table 3).

Table 4: Showing systolic blood pressure during various stages

\begin{tabular}{|l|c|c|c|}
\hline \multirow{2}{*}{ Time } & \multicolumn{2}{|c|}{ Systolic blood pressure } & \multirow{2}{*}{ p-value } \\
\cline { 2 - 3 } & Mean & S.D & \\
\hline Basal & $113.12 \pm$ & 14.13 & $0.001(<0.05)$ \\
\hline Induction 3 min & $103.58 \pm$ & 16.74 & $0.000(<0.05)$ \\
\hline Intubation 30 sec & 134.76 & 28.34 & $0.000(<0.05)$ \\
\hline Intubation 1 min & 138.10 & 23.97 & $0.000(<0.05)$ \\
\hline Intubation 3 min & 126.60 & 21.14 & \\
\hline
\end{tabular}




\begin{tabular}{|l|l|l|l|}
\hline Incision 1 min & 121.16 & 27.39 & $0.031(<0.05)$ \\
\hline Incision 3 min & 124.06 & 26.26 & $0.002(<0.05)$ \\
\hline Closure 5 min & 135.33 & 26.93 & $0.000(<0.05)$ \\
\hline Extubation 1 min & 145.44 & 23.57 & $0.000(<0.05)$ \\
\hline Extubation 15 min & 127.14 & 18.26 & $0.000(<0.05)$ \\
\hline
\end{tabular}

Table 5: Showing the diastolic blood pressure during various stages

\begin{tabular}{|l|c|c|c|}
\hline \multirow{2}{*}{ Time } & \multicolumn{2}{c|}{ Diastolic blood pressure } & \multirow{2}{*}{ p-value } \\
\cline { 2 - 3 } & Mean & S.D & \\
\hline Basal & 74.92 & 9.92 & \\
\hline Induction 3 min & 68.04 & 12.84 & $0.002(<0.05)$ \\
\hline Intubation 30 sec & 90.10 & 19.09 & $0.000(<0.05)$ \\
\hline Intubation 3 min & 84.44 & 15.11 & $0.000(<0.05)$ \\
\hline Incision 1 min & 79.64 & 18.02 & 0.065 \\
\hline Incision 3 min & 82.48 & 17.30 & $0.004(<0.05)$ \\
\hline Closure 5 min & 91.20 & 19.39 & $0.000(<0.05)$ \\
\hline Extubation 1 min & 95.06 & 15.59 & $0.000(<0.05)$ \\
\hline Extubation 15 min & 81.42 & 11.83 & $0.000(<0.05)$ \\
\hline
\end{tabular}

Both the systolic and diastolic blood pressure had a drop with induction while recovering at intubation and well maintained throughout the maintenance period and rise was observed at extubation. (Table 4 and 5)

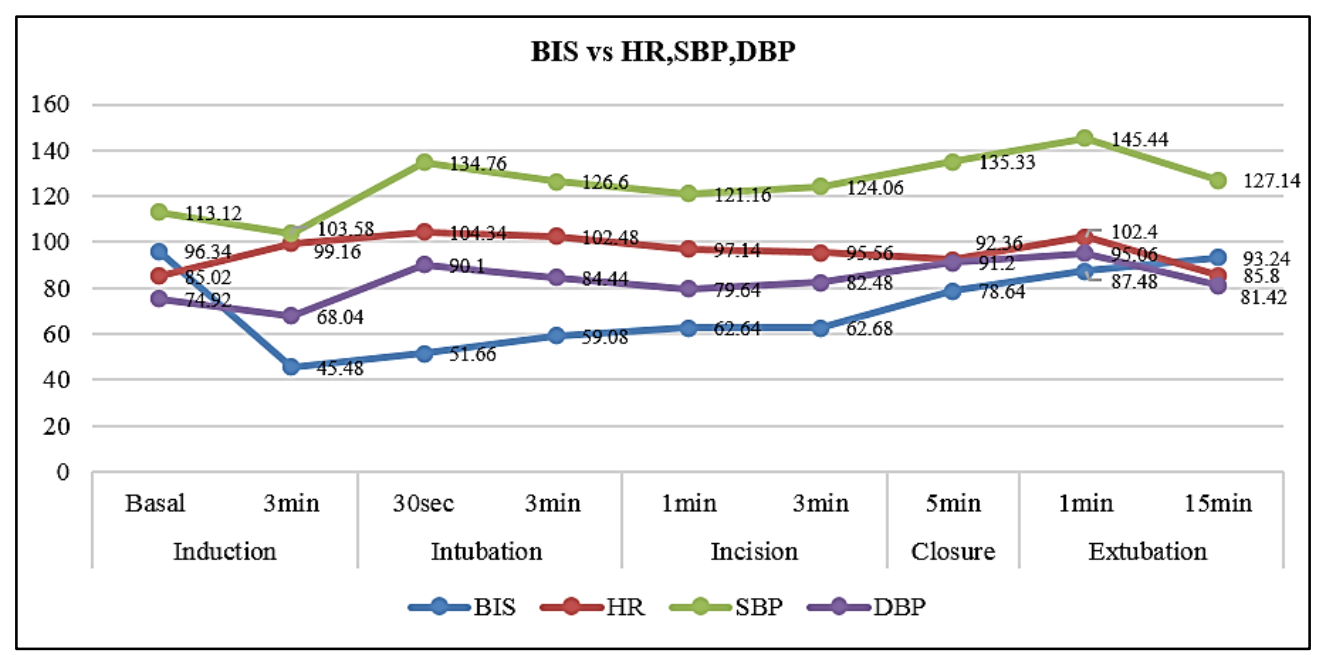

\section{Fig. 1: Comparison of BIS with hemodynamics}

BIS fell at induction along with SBP and DBP (except HR) and then a rise was observed at intubation and was well maintained throughout the anaesthesia maintenance and again rose to basal levels at extubation. The hemodynamics too correlated well with the rise and fall in BIS values (Fig. 1).

Table 6: Correlation of hemodynamic parameters and BIS values

\begin{tabular}{|l|c|c|c|c|c|}
\hline \multirow{2}{*}{ Time points } & \multicolumn{5}{|c|}{ Haemodynamics parameters and BIS values } \\
\cline { 2 - 6 } & $\begin{array}{c}\text { Heart rate } \\
(\mathbf{b p m})\end{array}$ & $\begin{array}{c}\text { SBP } \\
(\mathbf{m m} \text { Hg) }\end{array}$ & $\begin{array}{c}\text { DBP } \\
(\mathbf{m m ~ H g})\end{array}$ & $\begin{array}{c}\text { MAP } \\
(\mathbf{m m} \text { Hg) }\end{array}$ & SPO $_{\mathbf{2}} \%$ \\
\hline Basal & 0.118 & -0.080 & 0.059 & 0.064 & 0.209 \\
\hline Induction & & & & & \\
\hline $1 \mathrm{~min}$ & $0.334^{*}$ & -0.045 & -0.037 & 0.008 & $0.339^{*}$ \\
\hline $3 \mathrm{~min}$ & 0.152 & 0.050 & -0.014 & -0.010 & 0.128 \\
\hline $30 \mathrm{sec}$ & 0.154 & 0.231 & 0.174 & 0.182 & 0.072 \\
\hline Intubation & & & & & -0.037 \\
\hline 1 min & 0.113 & 0.084 & -0.120 & -0.055 & -0.136 \\
\hline $3 \mathrm{~min}$ & 0.104 & 0.242 & 0.175 & 0.207 & 0.191 \\
\hline 5 min & 0.200 & 0.143 & 0.104 & 0.093 & \\
\hline Incision & & & & & \\
\hline
\end{tabular}




\begin{tabular}{|l|c|c|c|c|c|}
\hline $1 \mathrm{~min}$ & -0.083 & -0.011 & -0.067 & -0.053 & $-0.301^{*}$ \\
\hline $3 \mathrm{~min}$ & 0.003 & -0.079 & -0.093 & -0.099 & 0.051 \\
\hline $5 \mathrm{~min}$ & 0.073 & -0.167 & $-0.340^{*}$ & -0.214 & 0.126 \\
\hline $10 \mathrm{~min}$ & 0.081 & $-0.363^{*}$ & $-0.312^{*}$ & $-0.316^{*}$ & -0.146 \\
\hline $15 \mathrm{~min}$ & 0.150 & -0.101 & -0.142 & -0.119 & 0.237 \\
\hline $20 \mathrm{~min}$ & 0.222 & $-0.286^{*}$ & $-0.282^{*}$ & -0.204 & 0.231 \\
\hline $25 \mathrm{~min}$ & 0.241 & -0.078 & -0.149 & -0.079 & 0.006 \\
\hline $30 \mathrm{~min}$ & 0.055 & 0.001 & -0.163 & -0.047 & $0.433^{* *}$ \\
\hline $35 \mathrm{~min}$ & -0.016 & -0.178 & -0.246 & -0.187 & 0.035 \\
\hline $40 \mathrm{~min}$ & 0.021 & -0.099 & $-0.299^{*}$ & -0.223 & $0.329^{*}$ \\
\hline Closure & & & & $-0.286^{*}$ & -0.089 \\
\hline $1 \mathrm{~min}$ & 0.124 & -0.205 & $-0.284^{*}$ & -0.271 & 0.076 \\
\hline $5 \mathrm{~min}$ & 0.009 & $-0.310^{*}$ & $-0.288^{*}$ & & 0.032 \\
\hline Extubation & & -0.050 & -0.139 & -0.229 & 0.080 \\
\hline $0 \mathrm{~min}$ & 0.067 & -0.116 & -0.202 & -0.161 & 0.215 \\
\hline $5 \mathrm{~min}$ & 0.276 & 0.160 & 0.194 & $0.300^{*}$ & \\
\hline $10 \mathrm{~min}$ & 0.044 & \multicolumn{2}{|l}{} &
\end{tabular}

Values are Pearson correlation (r values)

+ Suggestive of significance $(\mathrm{P}$ value: $0.05<\mathrm{P}<0.10)$

* Moderately significant (P value: $0.01<\mathrm{P} \leq 0.05$ )

$* *$ Strongly significant ( $\mathrm{P}$ value: $\mathrm{P} \leq 0.01$ )

Awareness under balanced anaesthesia over 24 hours period was nil in our study of 50 patients.

\section{Discussion}

Anesthetic drug effects have traditionally been measured by the observation of heart rate, blood pressure, breathing pattern and the presence or absence of movement. While these are useful measures, the cardiopulmonary effects of anesthetics are side effects, rather than direct indicators of the sedative and hypnotic effects, which are the main reason that anesthetics are given in the first place. Clearly, patients can experience intraoperative awareness in the absence of clinical signs of light anesthesia, such as changes in heart rate or blood pressure, or even movement. Therefore, a more direct and reliable method of measuring anesthetic drug effects on the brain is highly desirable and has been the object of research for many years. Electroencephalography (EEG) is an obvious brain monitoring modality because it is noninvasive and continuous. Many anesthetic drugs produce characteristic effects on the EEG, and these have been extensively studied. However, efforts to use EEG monitoring as a real time clinical tool were not successful until relatively recently. The development of relatively inexpensive, fast microcomputers was a critical prerequisite.

The results of this observational study show that BIS varies in various stages in general anaesthesia in response to the anesthetic agents used.

There was no statistically significant difference between the age, gender and weight of the patients except in one case who was 60-year-old who had an exaggerated fall in BIS to 22 on induction with propofol.

At induction with Inj. Propofol $2 \mathrm{mg} / \mathrm{kg}$, mean BIS decreased from a basal value of $96.34 \pm 1.7$ to $56.40 \pm 16.43$ and $45.48 \pm 10.31$ at $1^{\text {st }} \mathrm{min}$ and $3^{\text {rd }}$ min of induction respectively.
Flaishon et al., ${ }^{6}$ in their study used an intravenous injection of $4 \mathrm{mg} / \mathrm{kg}$ thiopental or $2 \mathrm{mg} / \mathrm{kg}$ propofol for $20 \mathrm{~s}$ for induction. The maximal induction agent effect (minimum BIS) was achieved $62 \pm 29 \mathrm{~s}$ after loss of consciousness with the propofol group and $43 \pm 16$ s in the thiopental group. Patients in the propofol group recovered consciousness $529 \pm 176$ s after induction and $421 \pm 175$ s after minimum BIS value. After induction, minimum BIS was significantly lower in the propofol group $(27 \pm 9)$ when compared with the thiopental group $(34 \pm 10)$. At the time of return of response to command,

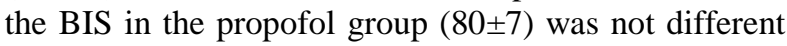
compared with thiopental $(81 \pm 5)$.

In our study, with intubation the BIS value increased to $51.66 \pm 12.44$ within 30 seconds from a post induction value of $45.48 \pm 10.31$. The BIS value further increased to $55.06 \pm 10.53$ and $59.08 \pm 9.82$ in the next $1 \mathrm{~min}$ and $3 \mathrm{~min}$ respectively.

Bruno Guignard et $\mathrm{al}^{7}$ in their study observed that BIS values were significantly $(\mathrm{P}<0.01)$ increased by laryngoscopy and orotracheal intubation in the remifentanil 0- and 2-ng/ml groups and that maximal increase in BIS was within 2 min of intubation in all cases.

After incision, the BIS score increased from $59.08 \pm 9.82$ to $62.64 \pm 10.48$ and $62.68 \pm 10.06$ at $1^{\text {st }}$ and $3^{\text {rd }}$ minute. This minimal increase in BIS could be because of the good depth of anaesthesia provided by the residual effects of propofol, analgesia by pentazocine and also because of the administration of inhalational agent.

During the maintenance phase in our study, BIS values were maintained between 50-70 until 15 min before closure where the BIS scores increased to 73-78 in the last $15 \mathrm{~min}$ to facilitate recovery from anaesthesia. 
This was brought about by stopping administration of inhalation agent at skin closure.

Gan $\mathrm{T} \mathrm{G}$ et $\mathrm{al}^{8}$ in their study maintained the BIS scores between 45-60 in the BIS monitored group and less than 50 in the standard practice group (where more propofol was used) and BIS was between 60-75 in the last 15 minutes of anaesthesia.

Mean BIS scores were slightly higher in our study. This could be due to presence of poor signal quality index and also because of use of halothane which is known to result in higher BIS scores when compared to other agents like sevoflurane and isoflurane.

Davidson $\mathrm{A} \mathrm{J}$ et $\mathrm{al}^{9}$ in their study showed that at 1 MAC, the BIS for halothane was significantly greater than isoflurane $(56.5 \pm 8.1$ vs $35.9 \pm 8.5, \mathrm{p}<0.0001)$. At awakening, there was no significant difference (BIS halothane; 81.1 (11.9), BIS isoflurane; 82.5 (6.4)

Immediate post extubation, the BIS values were $87.48 \pm 5.27$ and it increased to $93.24 \pm 2.82$ within 15 $\min$.

Gan T G et $\mathrm{al}^{8}$ in their study observed that patients in the BIS monitored group emerged from anaesthesia faster than the standard practice group. In the BIS monitored group, $43 \%$ of patients were fully oriented on arrival in the post-anaesthesia care unit compared with $23 \%$ in the standard practice group. The incidence of postoperative complications did not differ between groups.

In our study, there was initial increase in HR by 14 $\mathrm{bpm}$ at induction and then increased further to $20 \mathrm{bpm}$ coinciding with intubation which was statistically significant. The HR returned to baseline 10 min postincision. Post extubation, HR increased by $17 \mathrm{bpm}$ which was highly significant and returned to pre-operative value by $15 \mathrm{~min}$.

Systolic blood pressure decreased by $9 \mathrm{~mm} \mathrm{Hg}$ post induction and increased by $24 \mathrm{~mm} \mathrm{Hg}$ from preoperative value after intubation, both these values were statistically highly significant. Post extubation the pressures increased by $32 \mathrm{mmHg}$ which was highly significant.

Diastolic blood pressure fell by $6 \mathrm{~mm} \mathrm{Hg}$ by $3^{\text {rd }} \mathrm{min}$ of induction \& increased by $16 \mathrm{~mm} \mathrm{Hg}$ after $1 \mathrm{~min}$ of intubation which was statistically highly significant and again at extubation there was an increase by $20 \mathrm{~mm} \mathrm{Hg}$ which was statistically significant.

Though there is no statistical correlation between BIS scores and hemodynamic parameters, we can see that the Bispectral index varies with various stages of anaesthesia, almost simultaneous changes in systolic, diastolic and mean arterial blood pressures occurred. When there is a fall in blood pressure on induction, there is decrease in BIS scores, and on intubation, both the blood pressure as well as BIS increase. During maintenance phase heart rate, blood pressure and BIS are maintained at a constant level throughout the period and at extubation, there is again raise in the BIS scores along with the heart rate and blood pressure.
Kearse et al ${ }^{10}$ found a statistically significant difference between patients who mounted a haemodynamic response (BIS 67 \pm 10 ) compared with those who did not (BIS45 \pm 14 ). In this study, power spectral edge and median frequency did not distinguish those subjects who responded from those who did not.

Flaishon et $\mathrm{al}^{6}$ observed for thiopental, the heart rate at return of consciousness was significantly greater than that at $1 \mathrm{~min}$ before induction and at loss of consciousness. There were no other statistical differences in heart rate or blood pressure values in both propofol and thiopentone group at induction.

Guignard et $\mathrm{al}^{7}$ observed changes in BIS, MAP, and HR which were negatively correlated with remifentanil effect-site concentration ( $p<0.0001)$. Hypotensive episodes (MAP $<60 \mathrm{~mm} \mathrm{Hg}$ ) were noted in 1, 2, and 5 patients in the remifentanil $4 \mathrm{ng} / \mathrm{ml}, 8 \mathrm{ng} / \mathrm{ml}$, and 16 $\mathrm{ng} / \mathrm{ml}$ groups, respectively. We conclude that the addition of remifentanil to propofol affects BIS only when a painful stimulus is applied. Moreover, remifentanil attenuated or abolished increase in BIS and MAP after tracheal intubation in a comparable dosedependent fashion.

Atropine administered as a pre-medicant was associated with increase in heart rate. After administration of propofol, heart rate was maintained and there was fall in blood pressure noted. Increase in HR and BP at intubation in the present study indicates that better control would be possible with use of inhalational anaesthetics and analgesics to deepen the plane of anaesthesia.

There were exaggerated changes in the BIS scores and haemodynamics in few patients requiring changes in anaesthetic management. In one patient (60 years old), administration of $90 \mathrm{mg}$ of propofol resulted in a BIS score of 22 on induction, and to a fall in BP of $88 / 63 \mathrm{~mm}$ $\mathrm{Hg}$ with gradual improvement to a score of 36 in $3 \mathrm{~min}$ post intubation and BP of 74/50 mm Hg. Increase in IV fluid administration along with inj. mephentermine $6 \mathrm{mg}$ IV resulted in return to basal values with no further changes. The dose of propofol could have been exceeded initially resulting in above changes.

In another patient, in spite of adequate induction doses, BIS scores were at a higher value $(90,97)$ after 20 min of induction with well-maintained haemodynamics without obvious signs of lighter planes of anaesthesia. Propofol $20 \mathrm{mg}$ bolus IV was effective in bringing down the BIS, but it was consistently maintained at levels of 70 to 75 .

This type of response could be attributed to low signal quality index. Surprisingly, there was no incidence of awareness post operatively in this patient.

In 4 patients with significant hypertension and raised BIS score, deepening the plane of anaesthesia was effective. Among 2 patients, there were exaggerated increase in BP with well-maintained anaesthesia (as evidenced by BIS scores) necessitating treatment with vasodilators (nitroglycerine). 
One patient with tachycardia, hypertension and a BIS score of 67 was treated with bolus dose of inj. clonidine $60 \mathrm{mcg} \mathrm{IV}$, inhalational agents and a bolus dose of propofol $20 \mathrm{mg}$ IV.

There was no incidence of awareness at 24 hours noted in our study of 50 patients between 18 to 60 years under balanced anaesthesia technique.

Sebel PS et al ${ }^{11}$ conducted the first large prospective study examining the incidence of awareness during anaesthesia in the United States. Out of nearly 20,000 patients 25 patients experienced awareness, that is one person out of 783 or $0.13 \%$.

Sandin RH et al ${ }^{12}$ in their study of 11,785 patients, awareness occurred in $0.15 \%$ or ( 1 in 655 ).

Ekman et al., ${ }^{13}$ in their study observed two patients in the BIS-monitored group, $0.04 \%$ had explicit recall (ER) as compared with $0.18 \%$ in the control group $(\mathrm{P}<0.038)$. Both BIS-monitored patients with ER were aware during intubation when they had high BIS values (> 60) for 4 min and more than 10 minutes respectively.

Myles PS et a ${ }^{14}$ found that BIS monitoring lowered the risk of awareness by $82 \%$, in a group of patients at high risk for awareness during general anaesthesia.

Absence of awareness could be because of the small population studied, a short duration (24 hours) of observation and high-risk patients, surgeries with increased incidence of awareness were not included in our study.

The cost factor regarding the use of BIS for each patient for monitoring depth of anaesthesia to prevent the potential complication of post-traumatic stress disorder (PTSD) needs consideration.

Stand-alone BIS monitor costs at-least Rs.2.5 lakhs and each disposable electrode costs (approx) Rs.1500. This prohibitive cost is the limiting factor for its more frequent use. In addition, frequent improvements in the available versions as demanded by certain drawbacks associated with different sedative and inhalational agents will be an additional burden on the hospital.

The version used in the present study (version 1.0) has the ability to predict haemodynamic responses.

Subsequent versions of BIS (2.0 or 3.0) correlated significantly better with the Observer's Assessment of Awareness / Sedation (OAA/S), has very high prediction probability for correctly identifying loss of consciousness and predict responsiveness to verbal command during sedation or hypnosis better that either targeted or measured serum propofol concentration. Subsequently version 4.0 with better specificity and minimal EMG interferences is undergoing trials, pending release into the market.

Taken together, these data suggested that BIS accurately reflects the degree of sedation with volatile and intravenous hypnotic agent as well as predict physical movement.

\section{Conclusion}

Bispectral index is a simple, objective measure to assess depth of anaesthesia. There were predictable changes in BIS with the anaesthesia technique in the present study. Similarly, hemodynamic changes were also on the expected lines during the course of anaesthesia. However, the correlation between BIS and hemodynamics could not be statistically established. In view of significant disparities in BIS scores and hemodynamics in individual patients, anaesthesia management was suitably modified.

\section{References}

1. Domino KB. Closed malpractice claims for awareness during anaesthesia. ASA News letter, 1996;60:14-17.

2. Samuelsson P, Brudin L, Sandin R. Late psychological symptoms after awareness among consecutively included surgical patients. Anesthesiology. 2007;106: 26-32.

3. Moerman N, Bonke B, Oosting J. Awareness and recall during general anaesthesia. Facts and feelings. Anesthesiology. 1993;79:454-464.

4. Kaul. H L, Bharti N. Monitoring depth of anaesthesia. Indian J Anaesth. 2002;46(4):323-332.

5. Sigl JC, Chamoun NC. An introduction to bispectral analysis for the EEG. J Clin Monit. 1994;10(6):392-404.

6. Flaishon R, Windsor A. Sigl J, Sebel P. S. Recovery of Consciousness after Thiopental or Propofol: Bispectral Index and the Isolated Forearm Technique. Anesthesiology. 1997;3:613-619.

7. Guignard B, Menigaux C, Dupont X. The effect of remifentanil on the bispectral index change and hemodynamic response after orotracheal intubation. Anesth Analg. 2000;90(1):161-7.

8. Gan T J, Peter G S, Alastair W, Fredrick P, Carl R, Peter $\mathrm{S}$ et al. Bispectral index monitoring allows faster emergence and improved recovery from propofol, alfentanil and nitrous oxide anaesthesia. Anesthesiology. 1997;87:808-815.

9. Davidson AJ, Czarnecki C. The Bispectral Index in children: comparing isoflurane and halothane. $\mathrm{Br} J$ Anaesth. 2004;92:14-17.

10. Kearse L, Saini V, deBros F, Chamoun N. Bispectral analysis of EEG may predict anesthetic depth during narcotic induction. Anesthesiology. 1991;75(suppl 3A):A175.

11. Sebel PS, Bowdle A, Ghoneim M. The incidence of awareness during anaesthesia: A multicenter US study. Anesthesiology. 2003;99:A-360.

12. Sandin RH, Enlund G, Samuelsson P. Awareness during anaesthesia: A prospective study. Lancet. 2000;355:707.

13. Ekman A, Lindholm ML, Lennmarken C, Sandin R. Reduction in the incidence of awareness using BIS monitoring. Acta anaesthesiol Scand. 2004;48:20-6.

14. Myles PS, Leslie K, McNeil J, Forbes A, Chan MT. Bispectral index monitoring to prevent awareness during anaesthesia: The B-Aware Randomized Controlled Trial. Lancet. 2004;363(9423):1757-63.

How to cite this article: Rao GV, Chand $\mathrm{KN}$, Murthy AS, Balabhaskar S. Bispectral index analysis in general anesthesia: An observational study. Indian J Clin Anaesth. 2018;5(3):361-367. 